\title{
Expectativas para que México cambie el destino de sus exportaciones
}

\author{
LAgunas Puls, SERGIO \\ Departamento de Economía y Negocios \\ Universidad del Caribe (México) \\ Correo electrónico: slagunas@ucaribe.edu.mx \\ Cervantes Bello, Carmen Lilia \\ Departamento de Economía y Negocios \\ Universidad del Caribe (México) \\ Correo electrónico: ccervantes@ucaribe.edu.mx
}

\begin{abstract}
RESUMEN
En este artículo se analiza la exportación de México hacia la Unión Europea y China para conocer las probabilidades, en el caso de que las políticas comerciales de los Estados Unidos de América (EE.UU.) obligaran a cambiar el rumbo de los productos mexicanos hacia esos mercados. El trabajo se inicia con una síntesis de las políticas económicas de México hasta llegar a los acuerdos en materia comercial que tiene suscritos, con énfasis en el Tratado de Libre Comercio con la Unión Europea (TLCUEM) así como también esbozando la dinámica comercial de bienes con China. Posteriormente se describe trimestralmente la exportación mexicana de bienes y sus principales destinos, para después analizar los valores de la exportación desde el año 2012 y hasta el 2015, verificando que los datos se ajusten a distribuciones gamma y normal, estimando probabilidades para distintos ensayos, que contemplan los promedios exportados, los máximos e inclusive importes aún mayores para conocer las posibilidades que tendría México para sustituir distintas cantidades que eventualmente no pudieran colocarse en el mercado estadounidense.
\end{abstract}

Palabras clave: exportación mexicana, probabilidad, Unión Europea.

Clasificación JEL: F17; F15; C16; C13.

MSC2010: 6207; 60EXX; 60AXX. 


\title{
Expectations for Mexico to change the destination of its exports
}

\begin{abstract}
This article analyses the Mexican exports to China and the European Union along with the effects of a possible shift of Mexican products to the above markets due to a change within the US-Mexico trade agreements. Beginning with a review of Mexican trade agreements with the European Union and China, the article describes the quarterly Mexican exports of goods and products and their primary destinations to these countries. Later, the paper analyses the exports of Mexican products from years 2012-2015 fitting their values to Gamma and Normal distributions. Probabilities for different scenarios, which include the average and maximum exports, are given in order to quantify the possibilities that Mexico has to replace the goods and products that can not be positioned in the American market.
\end{abstract}

Keywords: Mexican export, probability, European Union.

JEL classification: F17; F15; C16; C13.

MSC2010: 6207; 60EXX; 60AXX.

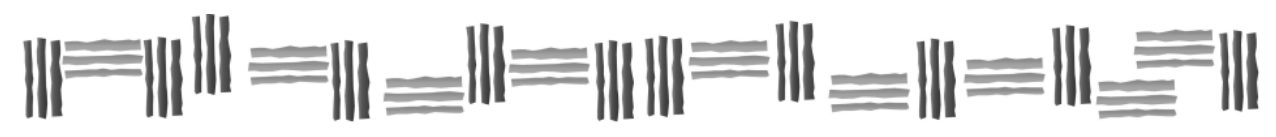




\section{Introducción.}

Durante algún tiempo prevaleció la idea de asociar el crecimiento económico con el auge del sector industrial, principalmente en la década de los ochenta y hasta finales del siglo XX. Quizás este fundamento tenga su origen en los efectos que la Revolución Industrial generó en los países desarrollados al desplazar la fuerza de trabajo concentrada en los sectores tradicionales como el agrícola, a las actividades de manufactura, que generaban más riqueza (Banco Interamericano de Desarrollo, 2010). Por su parte, la región de América Latina y el Caribe (ALC) intentó seguir la misma ruta hacia la industrialización. Sin embargo, los resultados obtenidos fueron poco exitosos, a pesar de que las políticas implementadas en los años ochenta en materia industrial consiguieron algunos logros, estos no fueron suficientes para poder competir a nivel internacional ya que los mercados resultaron demasiado pequeños (Lora \& Pagés, 2011).

Ante estos desafíos, una de las estrategias que adoptaron los países latinoamericanos fue la apertura comercial como pieza principal de la política exterior de la época, así como de los paquetes de liberalización y posterior ajuste estructural en gran parte de las economías de la región para afrontar los problemas de la deuda externa, la recesión, la desaceleración y la crisis económica de la década de los ochenta (Shearer \& Tres, 2016). Si bien, el camino hacia la integración y la reducción de aranceles fueron el foco de las discusiones en materia de comercio internacional en los noventa, actualmente las preocupaciones deberían ser, entre otras, la especialización humana y tecnológica.

En los últimos años, se ha podido observar un deterioro en los términos de intercambio, los tipos de cambio de la mayoría de los países de ALC han sufrido marcadas depreciaciones y aunque esto podría estimular el crecimiento de las ventas externas, la configuración actual de los tipos de cambio reales no favorece las perspectivas de expansión y diversificación de las exportaciones, lo que ha mermado su valor y generado déficits en la cuenta corriente de la balanza de pagos de los países (Giordano \& Ramos, 2016). De igual modo, es probable que los patrones de comercio global se vean modificados por la trayectoria de los acuerdos mega-regionales ${ }^{1}$ y la evolución de la dinámica de las Cadenas Globales de Valor (CGV). En términos generales, las economías latinoamericanas tienden a exportar materias primas no procesadas o de bajo valor agregado, situándose cerca del extremo inferior de las $\mathrm{CGV}^{2}$ debido en parte, a los elevados costos logísticos y de transporte y a sus bajos niveles de productividad (Blyde, 2014).

En cuestiones de geopolítica, de acuerdo con los escenarios previstos por el Atlantic Council (Marczak, Engelke, Bohl, \& Saldarriaga, 2016) para 2030, los patrones de comercio e inversión global serán multidireccionales, con diversos polos económicos importantes como EE.UU., Europa, China, India y un conjunto de potencias económicas de peso medio. No obstante, uno de los mayores riesgos es el aumento de la rivalidad entre Estados Unidos y China. La falta de solidez y coherencia en la política exterior estadounidense provocará tensiones entre ambas potencias, sobre todo en lo que concierne a los socios económicos y diplomáticos en ALC (Ramírez, 2014). Por lo tanto, este reacomodo obliga a la región a poner en marcha estrategias como la diversificación en destinos que minimicen los posibles efectos de tal interacción.

Adicionalmente, en materia de seguridad global, la corrupción, los efectos acumulados de las organizaciones criminales internacionales, el tráfico ilegal de personas, la protección

\footnotetext{
${ }^{1}$ Un acuerdo mega-regional se define como aquel que involucra a países que en su conjunto representan proporciones importantes de la población, el PIB, el comercio y la IED mundial y que además conlleva un fuerte compromiso en materia de reglamentación al interior de las fronteras, tales como el TTP, TTIP y RCEP. Ver INTAL Interactivo/El Mega-Regionalismo.

${ }^{2}$ La "curva de la sonrisa": Stan Shih describió la producción mundial como una U, en cuyos extremos se encuentran las actividades que agregan más valor como la investigación y el desarrollo, la creación de ideas, el branding y los contratos de servicios. En la base, se encuentran las actividades que agregan menor valor como la fabricación y el ensamblaje.
} 
ineficiente de los derechos de propiedad y el deficiente Estado de derecho a través de las instituciones débiles han cobrado su precio. La inseguridad económica y personal, acelera la fuga de cerebros y genera dificultades para atraer inversión extranjera directa, lo cual deriva en sobrecostos que disminuyen la competitividad de las empresas (Agosín, Fernández-Arias, Jaramillo, \& Lora, 2015).

Para el caso de México, después de registrar tasas de crecimiento cercanas al 7\% durante los años sesenta, el ingreso per cápita y los salarios reales se han estancado y la tasa de productividad solo ha experimentado un modesto aumento de $2 \%$ durante los últimos 50 años (Franco, 2016). Es cierto que algunos sectores de la economía mexicana son dinámicos gracias a las reformas efectuadas en décadas recientes para abrirlos al comercio y a la inversión. Sin embargo, otros sectores sufren de baja competitividad, en su mayoría debido al legado del corporativismo económico y el modelo político que sufrió el país durante gran parte del siglo XX (Vega, 2014). Estos datos de baja productividad y cifras de crecimiento económico reflejan mercados estancados, carencias de capital humano e insuficiente inversión pública, especialmente en infraestructura en comparación a otros países de desarrollo equiparable en situaciones iniciales similares como algunas economías asiáticas (Agosín, Fernández-Arias, Jaramillo, \& Lora, 2015).

A nivel internacional, la interacción entre México y EE.UU. ha estado marcada por la fuerte dependencia comercial al mercado estadounidense, debido en parte a la proximidad geográfica y si bien esto representa áreas de oportunidad también es fuente de riesgos considerables por la vulnerabilidad que genera esta relación casi exclusiva y fuertemente asimétrica. Si a esto se suma la llegada de Donald Trump a la presidencia y su postura tendente a la fragmentación, incluida la renegociación o extinción del Tratado de Libre Comercio de América del Norte (TLCAN) y el endurecimiento de la política migratoria, el panorama se torna árido.

En virtud de lo anterior, es necesario dar cuenta de las alternativas de exportación que tiene México, con especial interés en los mercados de Europa y China (mercados que actualmente ya figuran dentro de los principales destinos), en el caso de que las políticas comerciales de EE.UU. forzaran a cambiar el destino de los productos mexicanos por modificación e incluso posible cancelación del TLCAN, objetivo principal de este trabajo.

\section{Políticas Económicas.}

A continuación, se describe de manera breve la evolución de los modelos económicos aplicados por México en los últimos setenta años, desde el enfoque orientado al mercado interno y altamente proteccionista, hasta el neoliberalismo, poniendo especial énfasis en las acciones que se llevaron a cabo en materia de política comercial.

Desde mediados de los años cuarenta y hasta inicios de los ochenta, México siguió una estrategia de crecimiento basada en la industrialización por sustitución de importaciones (ISI). Se enfatizó en la necesidad de crear una importante base industrial con la intención de abandonar la dependencia del sector primario y fomentar la actividad de otras ramas mediante el aumento de la productividad de la mano de obra y el incremento del ahorro. Con una mayor relación capitalproducto en la economía se propuso ampliar el mercado interno y crear una base productiva exportadora y así poder captar las divisas que el país precisaba para su modernización, todo esto mediante la intervención directa del sector público (Monserrat \& Chávez, 2003). A pesar de que el modelo tuvo resultados positivos durante algún tiempo, comenzó a derrumbarse en los años setenta debido a la falta de capacidad tecnológica y financiera, deficiencias estructurales en la demanda y el alto grado de proteccionismo que prevaleció en la política comercial.

Ante esta muestra de debilidad en el crecimiento de la economía, se propuso el enfoque conocido como "Desarrollo Compartido" el cual transformó de manera radical la política fiscal y monetaria, manteniendo sin cambios significativos el resto de políticas. Con los ingresos obtenidos de la explotación y producción de los yacimientos petroleros descubiertos en esa época, 
se pretendió financiar la inversión en infraestructura productiva y orientar la producción hacia los bienes de capital (Cruz, 2017). Sin embargo, esto generó una gran dependencia hacia los recursos provenientes de las exportaciones petroleras, por lo que al caer los precios del petróleo en 1982 automáticamente se desplomó la captación de divisas, evidenciando así los desequilibrios estructurales de la economía junto con el déficit externo, el fiscal, la reducción del ahorro interno, la acumulación de rezagos productivos y un excesivo endeudamiento tanto interno como externo imposible de pagar (Torres \& Vega, 2010).

Ante esta precaria situación, el país requirió de una respuesta diferente por parte de las autoridades económicas y se vio forzado a negociar un rescate financiero con el Fondo Monetario Internacional (FMI), hecho que derivó en la implementación de una estricta política fiscal y monetaria, la desregulación del mercado, la privatización de la economía y una política comercial y de inversión tendiente a la apertura (Villareal, 2004). De este modo, la liberalización comercial da inicio en 1983 con una moderada reducción unilateral de los aranceles de importación y una gradual eliminación de los precios oficiales, así como de las cuotas, licencias y los permisos de importación. En 1986 México ingresa al Acuerdo General sobre Aranceles Aduaneros y Comercio (GATT) y para 1994 el arancel más alto a las importaciones era de 20\%, transformando al país en una de las economías más abiertas al mundo y atractiva para los inversionistas.

A pesar de estos indicadores positivos, la economía aún presentaba signos de vulnerabilidad y debilidad, puesto que había acumulado un creciente déficit en la cuenta corriente y la tasa de cambio semifija provocó una apreciación de las monedas extranjeras. Finalmente, la crisis del peso en 1995 provocó una recesión más profunda que en 1982; sin embargo, la recuperación fue mucho más rápida gracias al papel fundamental que jugó el comercio exterior (Ortiz, 2014).

\section{Tratados comerciales entre México, América del Norte y Europa.}

Durante la década de los noventa, México se convirtió en un fuerte impulsor del comercio internacional y mostró un notable interés en mantener una agenda de liberalización comercial que le permitiera tener acceso a los mercados del exterior y atraer inversión extranjera directa. En este sentido, la estrategia más proactiva que implementó fue la negociación de una red de acuerdos comerciales con distintas regiones del mundo siendo los más sobresalientes el TLCAN y el Tratado de Libre Comercio con la Unión Europea (TLCUEM), los cuales se abordarán a continuación.

Indudablemente el TLCAN ha sido uno de los acuerdos más importantes que ha suscrito el país y su trascendencia como modelo innovador de integración no tuvo que ver con su intención de ir en busca de una unión aduanera o un mercado común, sino más bien con el hecho de ser el primer tratado de desgravación arancelaria regional que incluyó cuestiones tan diversas e inéditas como: regulación sobre propiedad intelectual, legislación laboral y ambiental, comercio de servicios e inversión y mecanismos de solución de controversias ad hoc (Pastor, 2014). Con respecto a la liberación arancelaria, ésta se hizo adoptando un enfoque escalonado a un conjunto de categorías diferenciadas, proporcionando a cada una de ellas un marco temporal y una trayectoria progresiva hacia la eliminación completa de aranceles; mientras que el $75 \%$ de las líneas arancelarias se liberó durante el primer año, otras fueron sujetas a periodos de transición desde cinco y hasta quince años (Sehgal, 2010).

La decisión de negociar un acuerdo con EE.UU. y Canadá fue el resultado de una combinación de factores tanto internos como externos, ya que México promovió el acuerdo a fin de asegurar e impulsar el acceso de sus productos a uno de los mercados más importantes y así contrarrestar las prácticas proteccionistas de EE.UU. que en el pasado habían impedido el acceso a las exportaciones mexicanas competitivas (Vega, 2014). En este contexto, es importante señalar que los efectos de un área de libre comercio tardan un tiempo en manifestarse, ya sea por los ajustes estructurales que los países deben llevar a cabo como por la característica gradual incluida 
en el propio acuerdo. Sin embargo, es un hecho que el comercio entre México y EE.UU. ha crecido sustancialmente y de igual manera el capital externo que ha fluido al país ha contribuido a fortalecer a algunos sectores productivos como el industrial, destacándose la rama textil, automotriz y de productos eléctricos; por el contrario, otros sectores como el agrícola no han tenido el mismo dinamismo.

Ahora, si bien las relaciones con EE.UU. han sido una prioridad para México en materia de política económica internacional por el tamaño de su mercado, la proximidad geográfica y su papel hegemónico, estas mismas características han orillado al país a avanzar en una agenda de libre comercio más allá de América del Norte para dar continuidad a su política de apertura. Después de la entrada en vigor del TLCAN, la UE buscó establecer relaciones comerciales privilegiadas con México, ya que había demostrado tener la capacidad técnica y el compromiso político de integrarse con una gran potencia y sobre todo porque tenía el interés de recuperar su participación en el mercado mexicano que había estado disminuyendo a consecuencia del TLCAN (Bacaria, Osorio \& Artal, s.f.). De esta manera, en 1997 dan inicio las negociaciones del Acuerdo de Asociación Económica, Concertación Política y Cooperación también conocido como “Acuerdo Global” que si bien no contenía compromisos comerciales, sí contenía un mandato de negociación comercial predeterminado a través del Acuerdo Interino sobre Comercio y Cuestiones Relacionadas con el Comercio o TLCUEM (Curzio, 2004).

Las negociaciones entre México y la UE comenzaron en julio de 1998 y concluyeron después de 16 meses y nueve rondas. El alcance fue más allá de la desgravación arancelaria e incluyó diversos aspectos bajo un esquema de diálogo político, cooperación y comercio; la entrada en vigor del acuerdo se previó en dos etapas. La Decisión 2/2000 que cubre los temas de competencia exclusiva de la Comunidad Europea como el comercio de mercancías, compras públicas, disposiciones en materia de propiedad intelectual y de competencia, un mecanismo de solución de controversias y un dispositivo institucional. Por su parte, la Decisión 2/2001 cubre los temas que en su momento requerían ratificación por los Parlamentos nacionales de la UE como el comercio de servicios, inversiones, disposiciones adicionales sobre propiedad intelectual y solución de controversias (Frontini \& Bonnefoy, 2017).

A pesar de que el TLCAN fue uno de los acuerdos más innovadores, el TLCUEM es único en varios sentidos ya que es el más incluyente que haya negociado la UE con un solo país y el primero de una serie de acuerdos comerciales con países América Latina fuera del contexto de vecindad de la UE (Vega, 2011). Aun cuando EE.UU. probablemente continúe siendo el socio más importante para México y su mayor mercado de exportaciones, el TLCUEM representa una oportunidad para los exportadores mexicanos de expandir y diversificar sus ventas en el vasto y rico mercado de la UE.

No obstante, China seguirá avanzando en la modernización de su economía y esa transformación en marcha implica una oportunidad a considerar para México. Si bien, históricamente ALC no ha destacado en la política exterior china y los vínculos comerciales han sido poco equilibrados, estos se han consolidado sobre todo a principios de milenio, puesto que Chile, Costa Rica y Perú ya han suscrito acuerdos parciales y Colombia ha iniciado negociaciones (Jenkins, 2016). De igual manera, en la cumbre celebrada en 2015 entre China y la Comunidad de Estados Latinoamericanos y Caribeños (CELAC), se elaboró el plan de cooperación 2015$2019^{3}$ donde el gobierno chino se comprometió a otorgar US\$10 mil millones en préstamos para proyectos de infraestructura en la región, pero si bien, esta estrategia responde a los objetivos del gobierno chino y sus empresas, la relación estará condicionada en los próximos años por los avances que el gigante asiático logre con su reforma económica (Myers, 2016).

\footnotetext{
${ }^{3}$ Los esquemas $1+3+6$ y 3×3 respaldan la intención de fomentar una mayor participación de las empresas públicas y privadas chinas en los sectores industriales y de alta tecnología de América Latina, como son los de telecomunicaciones, logística, marítimo y ferroviario. Mientras que el modelo $3 \times 3$ pretende promover el traslado de cierta producción industrial china a América Latina.
} 
De acuerdo a lo anterior, en los siguientes apartados del trabajo se presenta el perfil de la exportación mexicana hacia Europa y China, con la finalidad de obtener probabilidades de ocurrencia para distintos importes que, derivados de medidas proteccionistas de los EE.UU. obligaran a buscar otros mercados destino, conociendo la opción con mayores posibilidades de éxito.

\section{Principales destinos de la exportación de bienes desde México.}

De acuerdo con el International Trade Centre (ITC), durante el año 2016 la exportación de bienes mexicanos hacia el mundo alcanzó 373,904,303 miles de dólares de los cuales el 81.02\% fue producto de las ventas a los EE.UU.; en segundo lugar se encontró Canadá con el 2.79\%, en tercera posición China alcanzando el 1.45\%, seguido por Alemania con el 1.06\%, Japón en el cuarto puesto representando $1.01 \%$ y en quinto lugar se colocó España logrando que ese mercado demandara $0.88 \%$ de productos mexicanos (International Trade Centre, 2017).

Gráfica 1. Los cinco destinos más importantes en la exportación de bienes desde México en 2016 (miles de dólares).

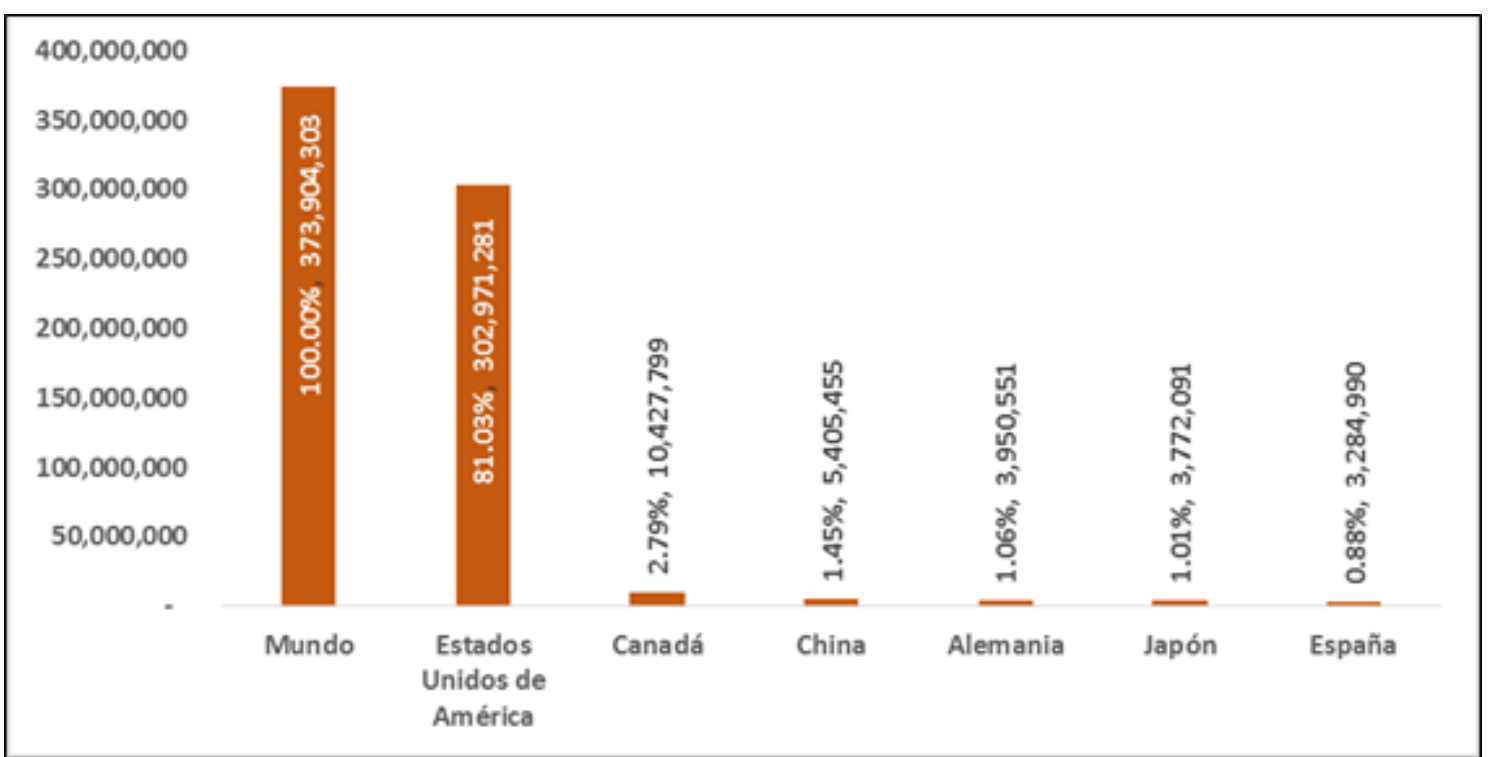

Fuente: Elaboración propia.

Para el caso de los bienes que constituyen la mayor exportación, de acuerdo con el Sistema de Clasificación Arancelaria y consultado en el ITC conforme a la cuantía al cierre del 2016, se sitúan en primer lugar los comprendidos en el Capítulo 87 con el $23.56 \%$ del total de las exportaciones; este capítulo agrupa bienes relacionados a vehículos automóviles, tractores, velocípedos y demás vehículos terrestres, sus partes y accesorios. En segundo lugar, se sitúan los bienes integrados en el Capítulo 85 que representan el 20.45\%, integrado por máquinas, aparatos y material eléctrico, y sus partes, aparatos de grabación o reproducción de sonido, aparatos de grabación o reproducción de imagen y sonido en televisión, y las partes y accesorios de estos aparatos. El tercer lugar con 16.50\% registra a los bienes comprendidos en el Capítulo 84 que son máquinas, aparatos y artefactos mecánicos, reactores nucleares, calderas, partes de estas máquinas o aparatos. El cuarto lugar está representado por el Capítulo 27 y constituye el 4.82\% de la exportación, integrando los combustibles minerales, aceites minerales y productos de su destilación, materias bituminosas y ceras minerales. El quinto puesto en importancia lo tiene el Capítulo 90 que agrupa instrumentos y aparatos de óptica, fotografía o cinematografía, de medida, control o precisión, instrumentos y aparatos médico-quirúrgicos, partes y accesorios de estos instrumentos o aparatos, un capítulo que representa el $4.34 \%$ del total de bienes exportados por México. 


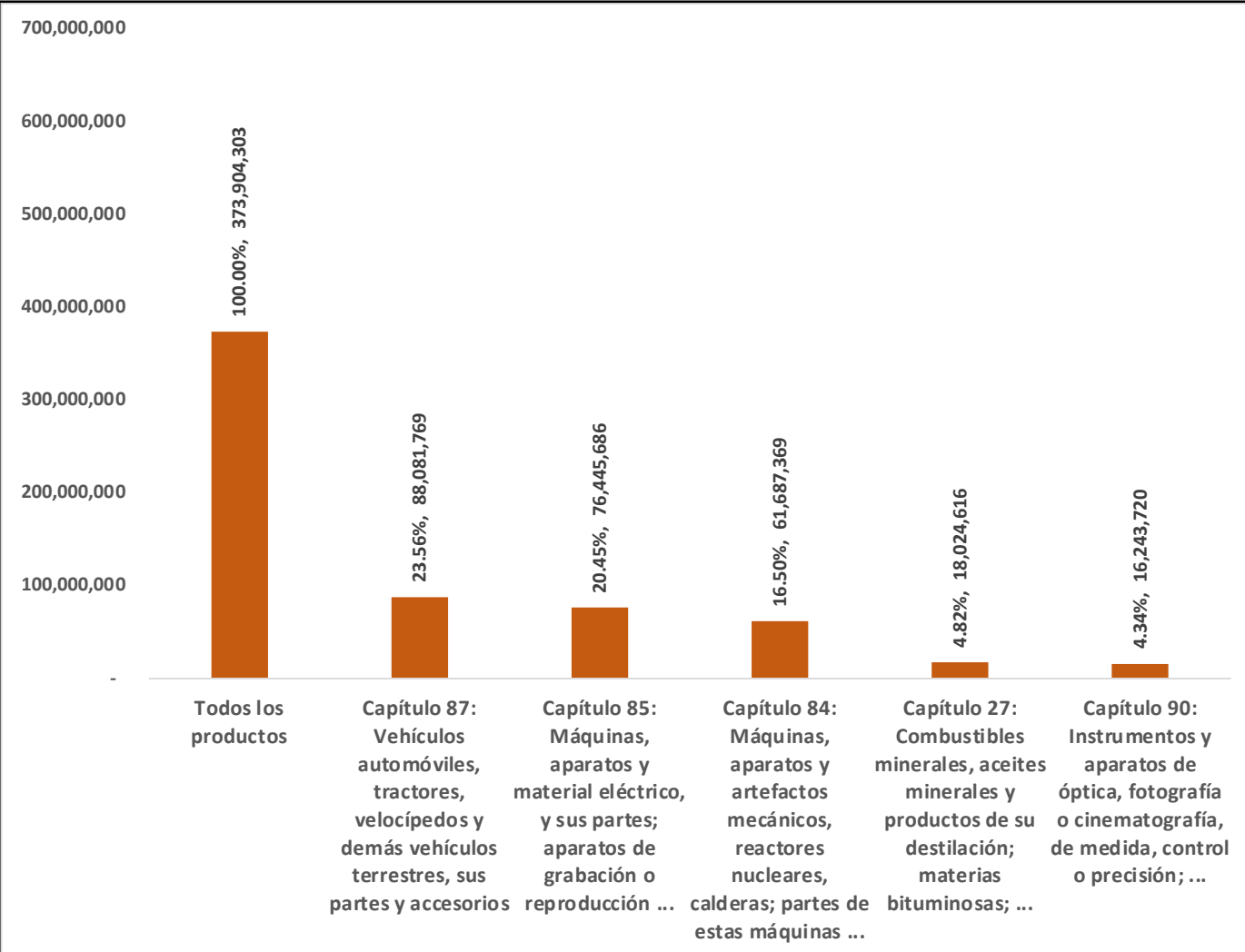

Fuente: Elaboración propia.

Como se aprecia en la Gráfica 2, la exportación contenida en los cinco principales capítulos ${ }^{4}$, representan en conjunto poco menos del $70 \%$ de toda la venta mexicana al mundo. Unido a lo anterior, si se acepta que México ha venido fortaleciéndose en determinados procesos productivos y de especialización, entonces resultaría una opción más inmediata procurar exportar aquello en lo que ya se tiene conocimiento y eficacia, en lugar de iniciar con nuevos procesos productivos sin saber incluso el rendimiento que se tendría, resultando entonces interesante, al menos de manera más inmediata, suplir mercados de los bienes consolidados.

Por lo anterior, en el siguiente apartado se tratará de responder la pregunta de investigación sustantiva, relativa a conocer cuáles son las probabilidades de que otros de los principales mercados de exportación puedan absorber distintas cantidades que no pudiera colocar México en los EE.UU., derivado de políticas económicas adversas que decidiera implementar el Presidente Donald Trump, como la posible cancelación del TLCAN, lo cual ha manifestado en más de una ocasión.

\section{Métodos de distribución gamma y normal.}

En este trabajo se emplean dos distribuciones de probabilidad, gamma (Khodabin \& Ahmadabadi, 2010) y normal (Wackerly, Mendenhall \& Scheafer, 2010), con la finalidad de conocer mediante ensayos, cuál sería la posibilidad de sustituir diferentes montos que por ajustes de políticas comerciales de los EE.UU. dificultaran la continuidad de la exportación mexicana, conociendo la probabilidad para remplazar el destino hacia China o la UE, considerando en este último caso a

\footnotetext{
${ }^{4}$ De acuerdo a los importes registrados en el año 2016, consultados en el International Trade Centre.
} 
todo el grupo de países que incluye a España y Alemania, clasificados dentro de los principales destinos de la exportación de México (PROMEXICO, 2017).

Mediante ambas distribuciones se analizan los valores, en dólares americanos, correspondientes a la exportación de México de los años 2001 a 2015; en el caso de la distribución gamma ésta se considera apropiada debido a que la exportación no se presenta con datos negativos y que, por la naturaleza propia de existencia de Tratados Comerciales, lo que se pretende es al menos conservar el promedio de exportación trimestral registrado hasta 2015, además de considerar una mesurada probabilidad para que se presenten incrementos considerables en la exportación por tratarse de mercados sustitutos (Arroyo, Bravo, Llinás \& Muñoz, 2014).

Por otra parte, se optó por analizar la información mediante la distribución de probabilidad normal para contrastar los resultados y no descartar que inclusive, con este tipo de distribución, pudieran presentarse, a diferencia de la forma gamma, disminuciones del promedio exportado.

Para ambos métodos se aplicó la prueba Anderson - Darling (Pomory, 2006) con valor crítico (Ang \& Willson, 2007) de 0.751 así como también prueba visual mediante gráfica de probabilidad.

La distribución gamma, representada por la Gráfica 3, está definida por una escala $(\beta)$ y una forma $(\alpha)$ que le otorga una figura con sesgo positivo.

Gráfica 3. Representación de una distribución gamma.

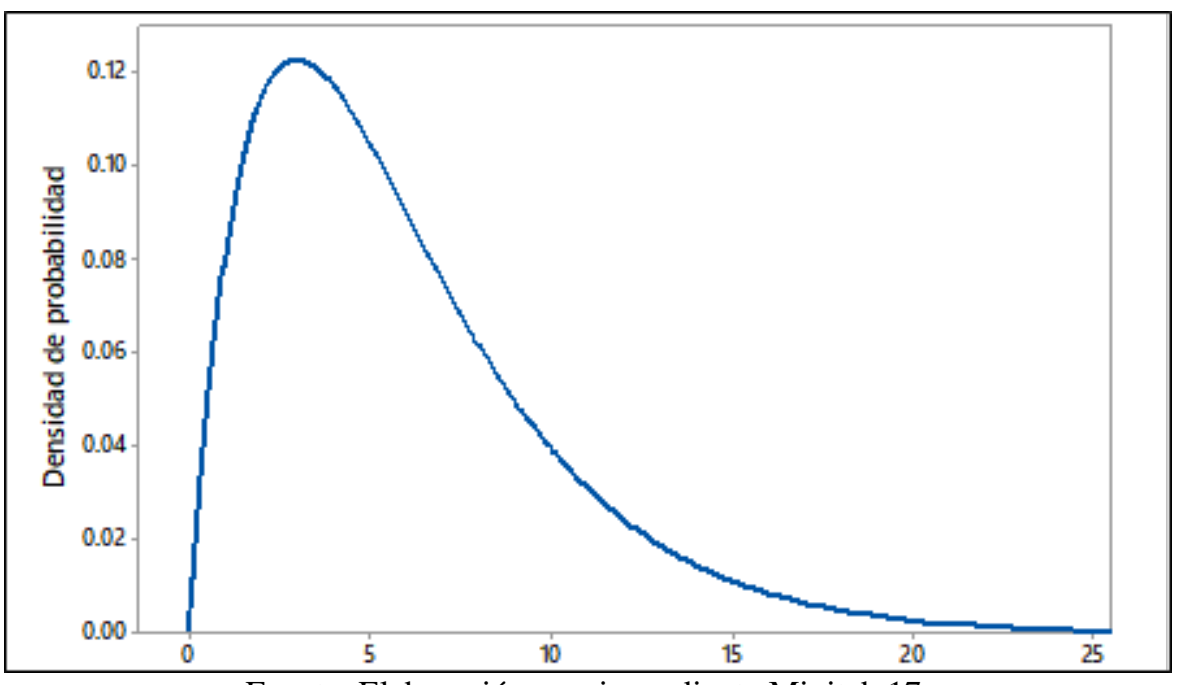

Fuente: Elaboración propia mediante Minitab 17.

En el eje horizontal se presentarán importes de la exportación que se analice en cada caso; en cuanto a los parámetros gamma se estará a lo siguiente (Thom, 1958):

$$
\begin{gathered}
A=\ln \mu-\frac{\sum_{i=i}^{n} \ln x_{i}}{n} \\
\alpha=\frac{1}{4 A}\left[1+\sqrt{\left(1+\frac{4 A}{3}\right)}\right] \\
\beta=\frac{\mu}{\alpha}
\end{gathered}
$$


En lo que se refiere al método de distribución normal representado por la Gráfica 4, los parámetros necesarios para la obtención de probabilidades, así como la representación gráfica son la media $(\mu)$, la desviación estándar $(\sigma)$ y la varianza $\left(\sigma^{2}\right)$.

$$
\begin{aligned}
& \mu=\frac{\sum_{i=1}^{n} x_{i}}{n} \\
& \sigma^{2}=\frac{\sum_{i=1}^{n}\left(x_{i}-\mu\right)^{2}}{n-1} \\
& \sigma=\sqrt{\sigma^{2}}
\end{aligned}
$$

Gráfica 4. Representación de una distribución normal.

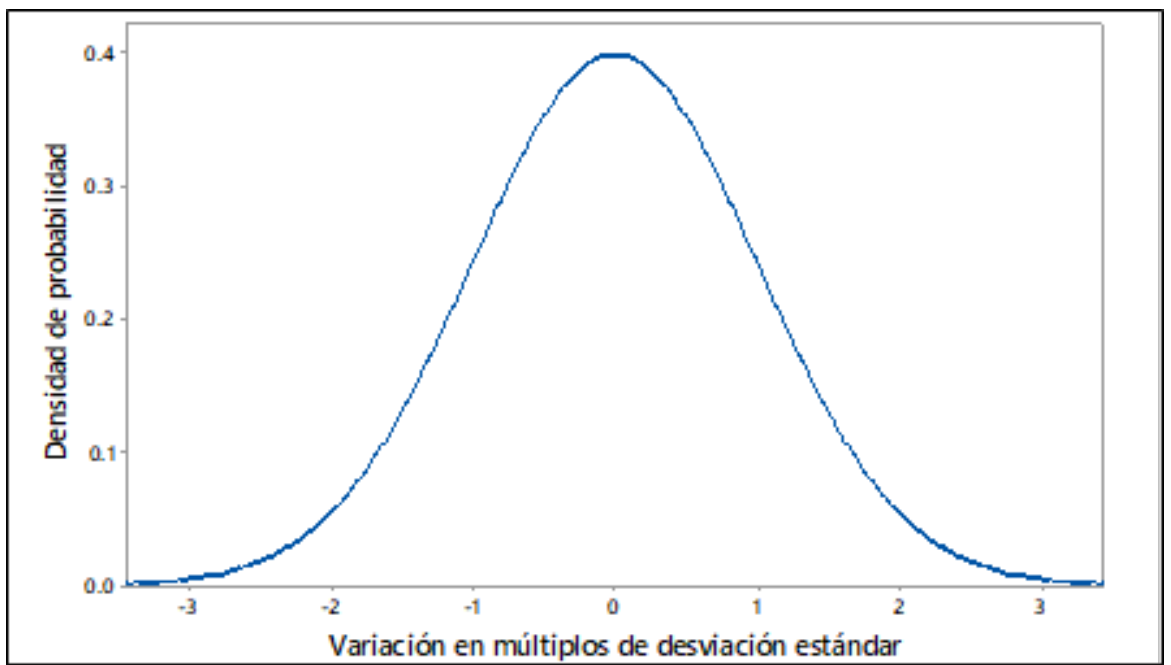

Fuente: Elaboración propia mediante Minitab 17.

Mediante los dos métodos descritos anteriormente se estimarán probabilidades de ocurrencia para que la exportación de México ya sea hacia la UE o China, pudiera lograr colocarse en esos mercados. Lo anterior se presenta a manera de ensayos, cada uno con importes de exportación, en dólares americanos, de la forma “desde y en adelante” lo que permitirá obtener la probabilidad formal de sustituir exportación. La información que se analiza es por cuatrimestres, en virtud de analizar un mayor número de datos y no solamente la concentración anual, iniciando en el primer cuatrimestre del año 2012 y hasta el cuarto trimestre del $2016^{5}$.

\section{Parámetros y probabilidades para el mercado de la Unión Europea.}

A continuación, se presentan distintos ensayos, con variantes en rangos de exportación que eventualmente necesitara México colocar debido a condiciones comerciales adversas en los EE.UU. La Gráfica 5 concentra la exportación colocada en el mercado de la UE y servirá para obtener los estadísticos necesarios para los dos métodos de análisis adoptados.

\footnotetext{
${ }^{5}$ Los análisis del apartado siguiente no analizan la exportación hacia los EE.UU. ya que el interés es en conocer las probabilidades hacia mercados alternativos.
} 
Gráfica 5. Exportación mexicana de bienes hacia la Unión Europea (miles de dólares).

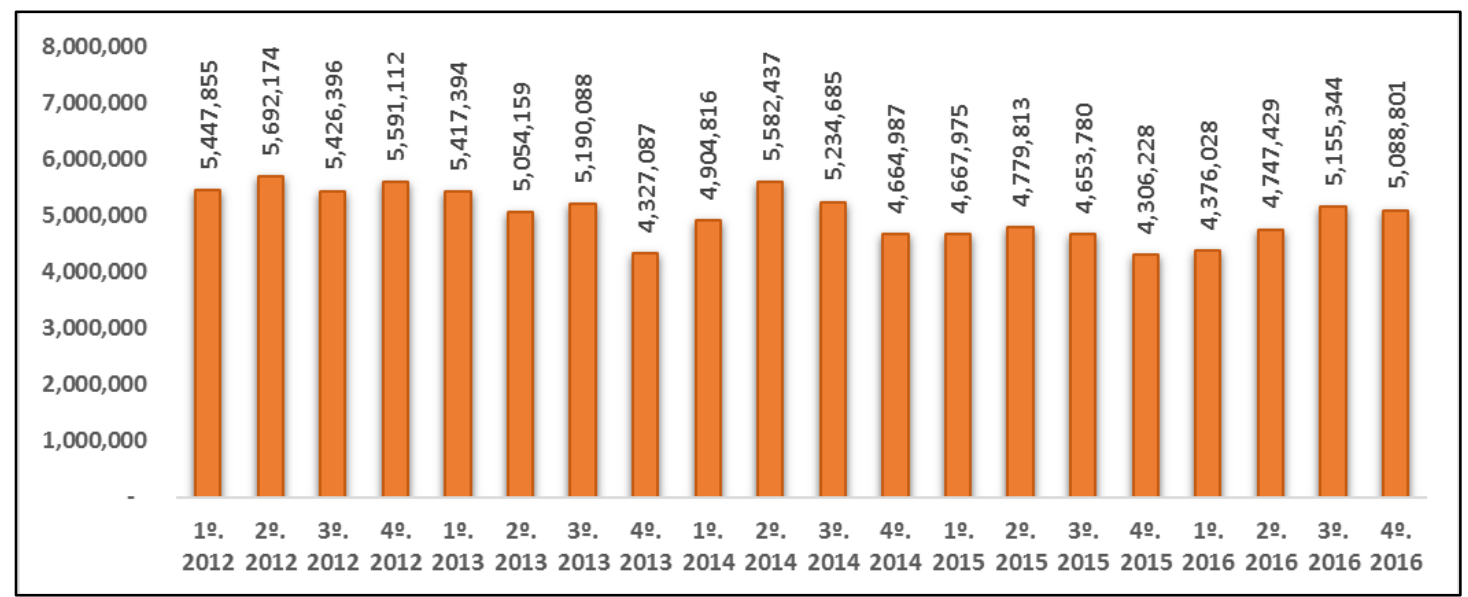

Fuente: Elaboración propia.

Empleando las ecuaciones (1), (2) y (3) se obtienen los siguientes parámetros para la distribución gamma:

$$
\begin{gathered}
A=\ln \mu-\frac{\sum_{i=i}^{n} \ln x_{i}}{n}=15.428-\frac{308.487}{20}=0.004 \\
\alpha=\frac{1}{4 A}\left[1+\sqrt{\left(1+\frac{4 A}{3}\right)}\right]=\frac{1}{(4 * 0.004)}\left[1+\sqrt{\left(1+\frac{(4 * 0.004)}{3}\right)}\right]=137.078 \\
\beta=\frac{\mu}{\alpha}=\frac{5,015,429}{137.08}=36,588.606
\end{gathered}
$$

En cuanto a los parámetros para la distribución normal, las ecuaciones (4), (5) y (6) arrojaron los siguientes resultados:

$$
\begin{gathered}
\mu=\frac{\sum_{i=1}^{n} x_{i}}{n}=\frac{100,308,588}{20}=5,015,429.40 \\
\sigma^{2}=\frac{\sum_{i=1}^{n}\left(x_{i}-\mu\right)^{2}}{n-1}=\frac{3,632,905,813,763}{19}=191,205,569,145.421 \\
\sigma=\sqrt{\sigma^{2}}=\sqrt{191,205,569,145.421}=437,270.590
\end{gathered}
$$

A partir de los resultados anteriores se hace posible realizar la prueba de bondad y ajuste, específicamente el test conocido como Anderson-Darling ${ }^{6}$, prueba que permitirá comprobar si los datos siguen una distribución gamma y normal, situación que se presentará siempre que el resultado obtenido para AD (Anderson \& Darlin, 1952) no supere el valor crítico adoptado para un nivel de confianza del 95\%.

Por lo anterior, la hipótesis para aceptar que los datos pueden ser analizados con ambas distribuciones será:

Si $A D<0.751$ se acepta analizar la exportación con los métodos propuestos, si no se rechaza

\footnotetext{
${ }^{6}$ Esta prueba es también conocida en diversos textos como $A^{2}$ desde el análisis realizado por Stephens (1979a).
} 
La ecuación para obtener AD (Stephens, 1979b) se presenta a continuación:

$$
A D=-\frac{\left\{\sum_{i=1}^{n}(2 i-1)\left[\ln z_{i}+\ln \left(1-z_{n+1-i}\right)\right]\right\}}{n}-n
$$

Con la ecuación 7 y los parámetros previamente calculados para la exportación hacia la UE, se obtuvo lo siguiente:

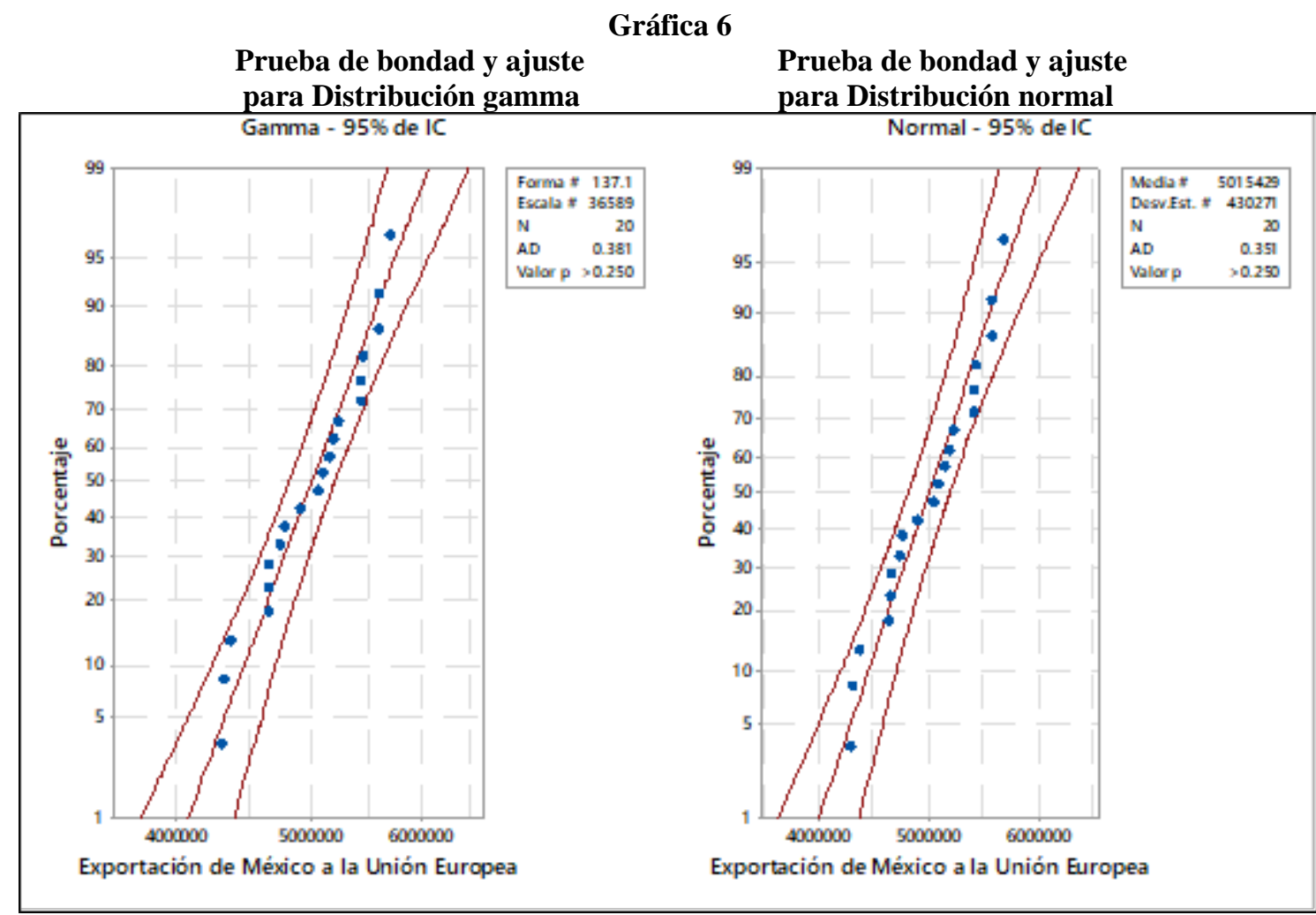

Fuente: Elaboración propia mediante Minitab 17.

Los resultados para la prueba AD que se presentan en la Gráfica 6 permiten aceptar los métodos de distribución adoptados, para gamma $0.381<0.751$ y para la distribución normal fue $0.351<0.751$ con base a lo anterior, se procede a calcular las probabilidades para montos de exportación iniciando con el mayor importe registrado y a partir del mismo con incrementos de 25,000 (también miles de dólares) para contrastar los resultados con respecto a China. Los estimados se presentan en la Tabla $1^{7}$.

\footnotetext{
${ }^{7}$ La Tabla 1, en su primer renglón considera, señalada con un asterisco, la exportación trimestral promedio, posteriormente se va incrementando el importe para cada renglón a razón de 50,000,000 de dólares (la tabla expresa cifras en miles); la cantidad señalada con dos asteriscos representa la máxima exportación registrada en un trimestre que en los siguientes renglones se va incrementando también a razón de 50,000,000 dólares para conocer la probabilidad de que el mercado de la UE pueda adquirir más mercancía desde México.
} 
Tabla 1. Parámetros y probabilidades para el mercado de China.

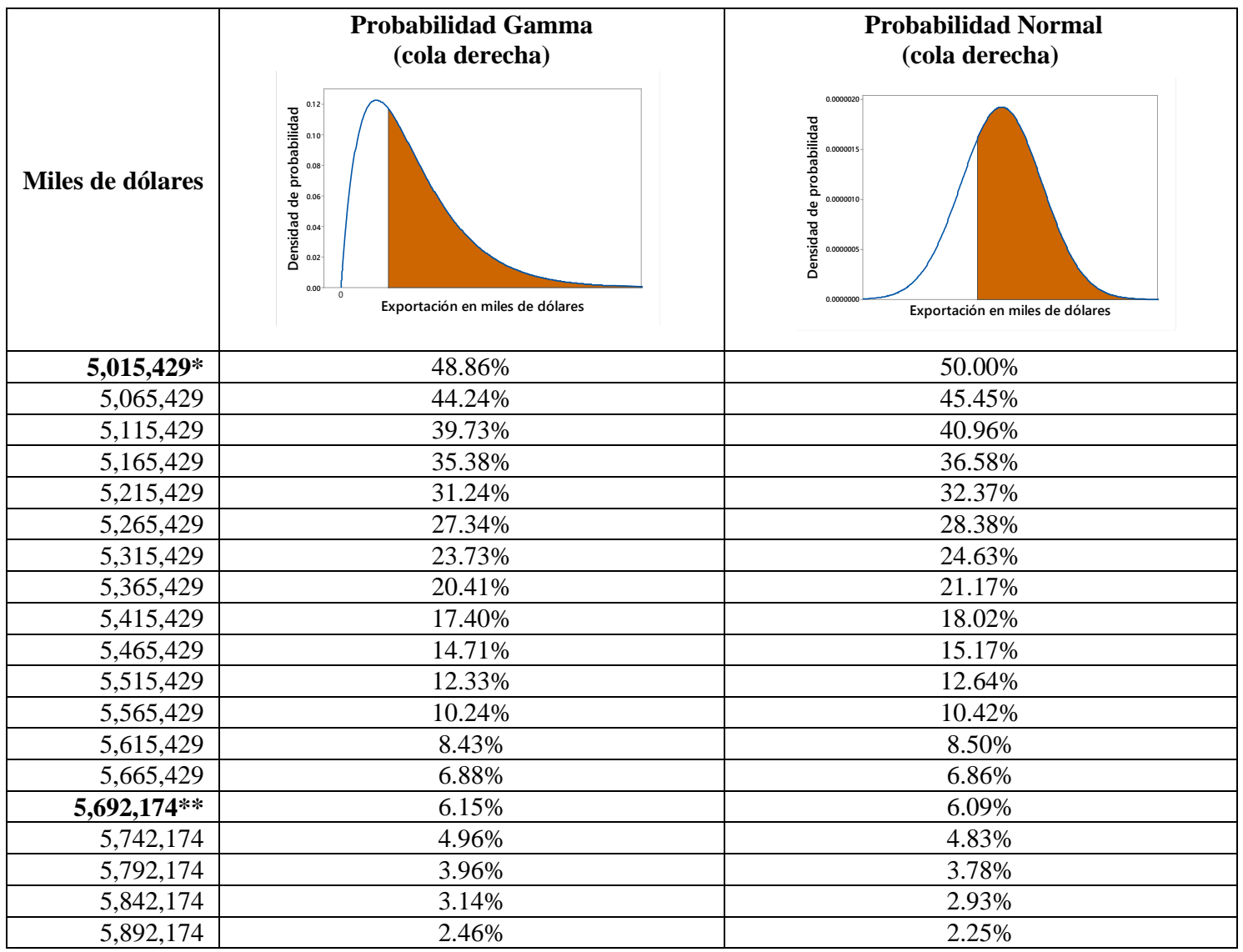

Nota*: representa el promedio de la exportación cuatrimestral de la información analizada.

Nota**: representa la cantidad máxima exportada en un trimestre de la información analizada. Fuente: Elaboración propia.

Conservando la misma metodología descrita anteriormente, a continuación, se presenta la Gráfica 7 que concentra la exportación de México hacia China, iniciando en 2012 y hasta el último trimestre del 2016.

Gráfica 7. Exportación mexicana de bienes hacia China (miles de dólares).

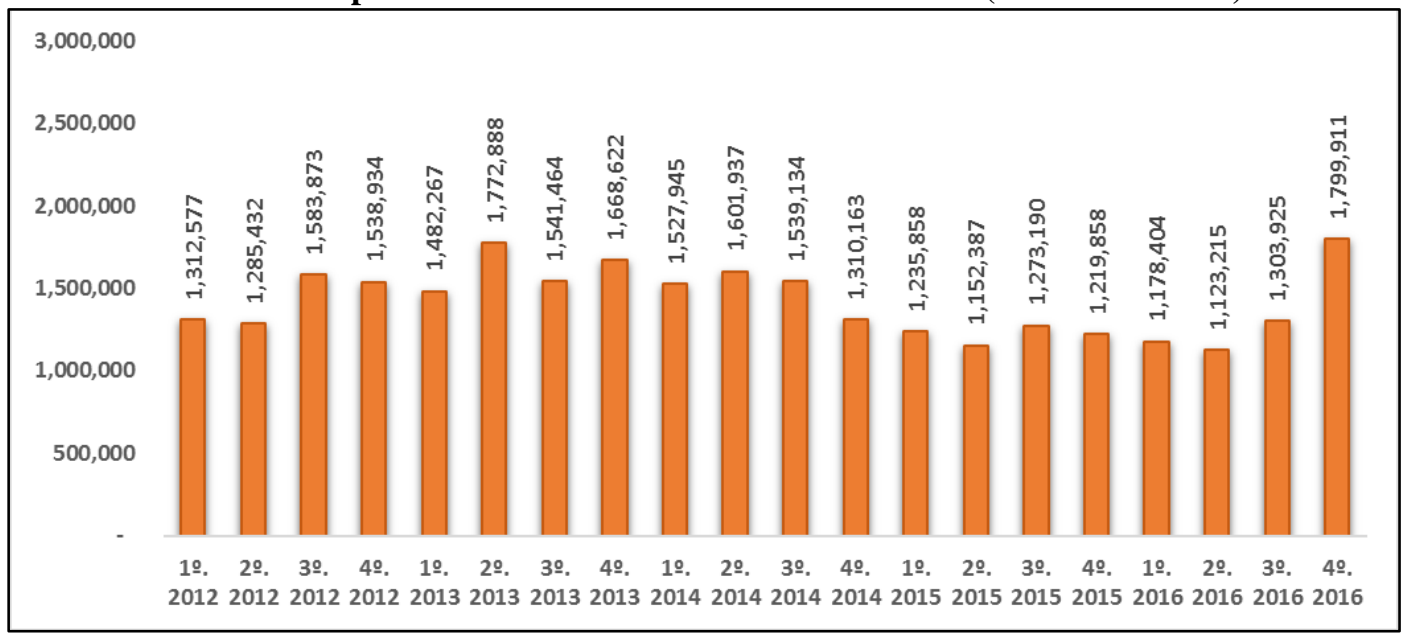

Fuente: Elaboración propia.

Empleando las ecuaciones (1), (2) y (3) se obtienen los siguientes parámetros para la distribución gamma: 


$$
\begin{gathered}
A=\ln \mu-\frac{\sum_{i=i}^{n} \ln x_{i}}{n}=14.168-\frac{283.159}{20}=0.010 \\
\alpha=\frac{1}{4 A}\left[1+\sqrt{\left(1+\frac{4 A}{3}\right)}\right]=\frac{1}{(4 * 0.010)}\left[1+\sqrt{\left(1+\frac{(4 * 0.010)}{3}\right)}\right]=50.270 \\
\beta=\frac{\mu}{\alpha}=\frac{1,422,599}{50.27}=28,299.165
\end{gathered}
$$

En cuanto a los parámetros para la distribución normal, las ecuaciones (4), (5) y (6) arrojaron los siguientes resultados:

$$
\begin{gathered}
\mu=\frac{\sum_{i=1}^{n} x_{i}}{n}=\frac{28,451,984}{20}=1,422,599.20 \\
\sigma^{2}=\frac{\sum_{i=1}^{n}\left(x_{i}-\mu\right)^{2}}{n-1}=\frac{817,887,307,941}{19}=43,046,700,417.947 \\
\sigma=\sqrt{\sigma^{2}}=\sqrt{43,046,700,417.947}=207,476.987
\end{gathered}
$$

Con los estimados anteriores, se aplica la prueba de bondad y ajuste AD mediante la ecuación (7), presentando los resultados en la Gráfica 8.

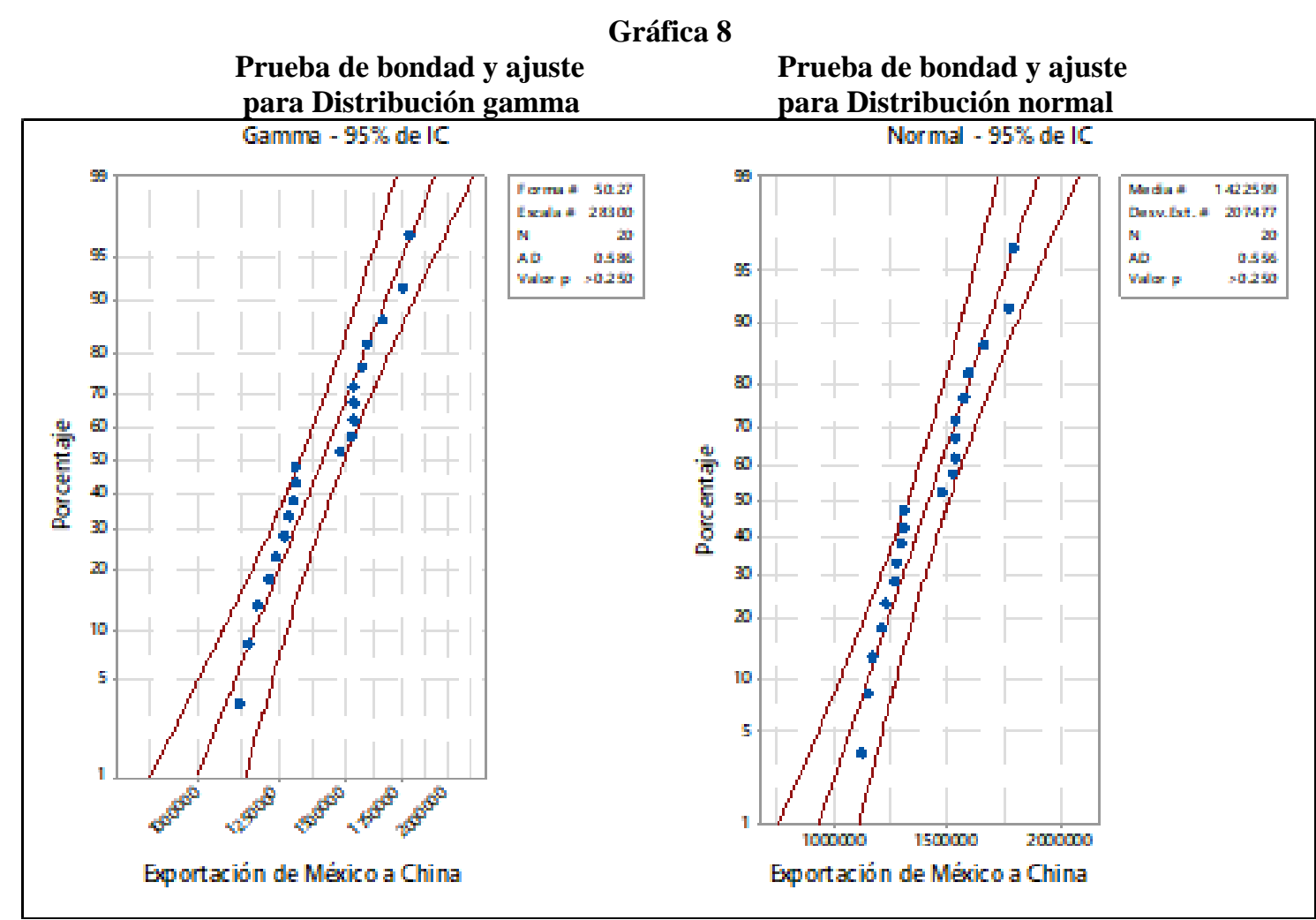

Fuente: Elaboración propia mediante Minitab 17.

Conforme a la Gráfica 8, la prueba AD resultó menor al valor crítico, para distribución gamma $0.586<0.751$ por lo que se acepta como método para obtención de probabilidades; en el caso de la distribución normal resultó $0.556<0.751$ por lo que también es un método de distribución aceptado para obtención de probabilidades. 
A continuación, se presenta la Tabla $2^{8}$ considerando diferentes importes para la exportación desde México a China:

Tabla 2. Exportación a China desde y en adelante (miles de dólares).

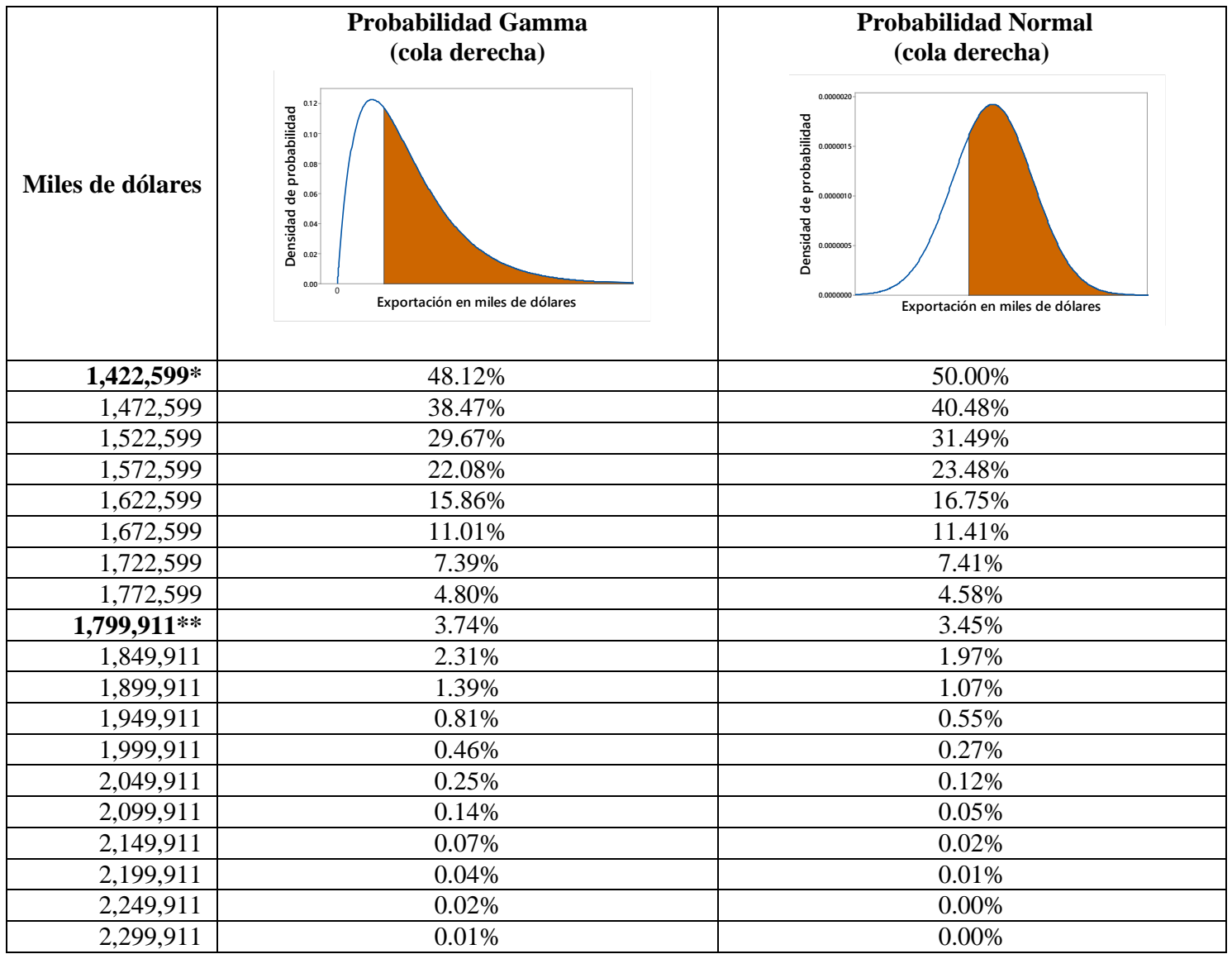

Nota*: representa el promedio de la exportación cuatrimestral de la información analizada.

Nota**$^{* *}$ representa la cantidad máxima exportada en un trimestre de la información analizada.

Fuente: Elaboración propia.

\section{Resultados obtenidos.}

Para cada uno de los mercados analizados se inició en un primer ensayo con el promedio que México exportó en un trimestre, desde 2012 y hasta 2015 agregando en los siguientes renglones o ensayos \$50,000,000 de dólares, que representan cantidades que eventualmente necesitara cubrir México debido a políticas comerciales proteccionistas de los EE.UU., las probabilidades obtenidas para continuar exportando al menos el promedio trimestral. En el caso de la UE fue de 48.86\% en distribución gamma y como era de esperar del 50\% para distribución normal; en cuanto a China se obtuvo que el promedio de la exportación puede permanecer para un siguiente trimestre en $48.12 \%$ mediante distribución gamma y el 50\% por distribución normal.

Al incrementar la exportación a distintos niveles se observa que, por la característica de la distribución gamma con sesgo positivo, las probabilidades para que cada mercado pudiera importar más productos desde México fueron mayores en este tipo de distribución que las

\footnotetext{
${ }^{8}$ La Tabla 2, en su primer renglón considera, señalada con un asterisco, la exportación trimestral promedio, posteriormente se va incrementando el importe para cada renglón a razón de 50,000,000 de dólares (la tabla expresa cifras en miles); la cantidad señalada con dos asteriscos representa la máxima exportación registrada en un trimestre la cual, en los siguientes renglones se va incrementando también a razón de 50,000,000 dólares para conocer la probabilidad de que el mercado de la UE pueda adquirir más mercancía desde México.
} 
obtenidas por distribución normal, por ello en los renglones que representan la mayor exportación registrada en un trimestre, en el caso de la UE que alcanzó la cantidad de \$5,692,174,000 dólares, el que se volviera a registrar exportación a partir de esa cantidad, tendría una probabilidad de $6.15 \%$ por distribución gamma y de $6.09 \%$ por distribución normal; en cuanto a los resultados para que China continúe exportando su máximo trimestral registrado de \$1,799,911,000 o más, alcanzó 3.74\% por gamma y 3.45\% mediante distribución normal.

Ahora bien, al incrementar las cantidades máximas hasta ahora registradas en un trimestre, en los ensayos en que se aumentaron inicialmente \$50,000,000 de dólares al máximo histórico, resultó que, para que la UE importara de México a partir de \$5,742,174,000 dólares, alcanzaría mediante gamma la probabilidad de $4.96 \%$ y mediante distribución normal solo $4.83 \%$. En cuanto a China, el primer agregado de \$50,000,000 dólares a su máximo histórico trimestral, arrojó que para que importara desde México una cantidad a partir de \$1,849,911,00 dólares, la probabilidad gamma sería de $2.31 \%$ y en distribución normal de solo el $1.97 \%$.

En los siguientes ensayos que implicaron agregar a los máximos históricos cantidades desde $\$ 100,000,000$ de dólares, las probabilidades descienden gradualmente, iniciando para la UE en 3.96\% y 3.78\% para cada distribución, pretendiendo exportar a partir de \$5,792,174,000 y en cuanto al análisis del mercado de China se podría esperar probabilidades de $1.39 \%$ para gamma y de $1.07 \%$ en normal para que importara \$1,899,911,000 dólares; en los siguientes ensayos con mayores incrementos, las probabilidades obtenidas para ambas distribuciones disminuyeron hasta prácticamente cero.

\section{Conclusiones.}

Después de observar la clara dirección de las exportaciones de bienes desde México hacia el mundo, es y ha sido clara la dependencia hacia ciertos destinos, entre estos EE.UU., Canadá, países de la UE y China. No obstante, después de más de 20 años, al estar en riesgo las ventajas del TLCAN por cambios en políticas comerciales de la administración del Presidente Donald Trump, que incluso pudieran llevar a la cancelación del TLCAN, provoca que de manera recurrente surjan opiniones acerca de la urgente necesidad de México para diversificar su actual colocación de bienes en el extranjero, procurando obtener mejor provecho de todas las condiciones comerciales con otros países o regiones, nombrando entre éstas opiniones a la UE, por el amplio tratado comercial que se tiene desde el año 2000 y a China, por su gran dinamismo y crecimiento económico.

Por lo anterior, al desarrollar este trabajo se buscó atender el objetivo general, estimando las probabilidades para conocer, en caso de que las políticas comerciales de EE.UU. obligaran a cambiar el destino de los productos mexicanos, con cuántas posibilidades formalmente estimadas, se podría realmente argumentar que ya fuese hacia la UE o hacia China, México lograra colocar, además de las cantidades de exportación hasta ahora observadas, un mayor importe en millones de dólares, iniciando con \$50,000,000 de dólares adicionales a partir del promedio trimestral e inclusive, por encima del máximo importe registrado en un trimestre. Pudiendo decir que los resultados no son precisamente halagadores ya que tan solo en pensar conservar los promedios de exportación, para ambos casos analizados y como era de esperar, las probabilidades se encuentran cercanas al $50 \%$.

Pero quizá lo que refleje una mayor urgencia de atención es que sin duda, de requerir fortalecer un mercado destino para las exportaciones mexicanas, éste deberá ser Europa por dos razones sustanciales. La primera es que es un mercado con Tratado en materia comercial vigente y en proceso de consolidación pero, además, las probabilidades obtenidas para lograr consolidar una mayor cantidad de exportación son mucho más convenientes que con respecto a China, tan solo pensar en la posibilidad para continuar exportando a partir del máximo importe trimestral registrado en la UE que alcanzó \$5,692,174,000 dólares, se tendrían probabilidades de entre 6.09\% mediante distribución gamma y de 6.15\% por distribución normal. 


\section{Referencias}

Agosín, M., Fernández-Arias, E., Jaramillo, F., \& Lora, E. (2015). Realidad Macroeconómica Latinoamericana. Módulo I. Las restricciones a la inversión privada y el crecimiento. USA: Banco Interamericano de Desarrollo. Recuperado de

https://indesvirtual.iadb.org/pluginfile.php/45725/mod_resource/content/1/MOD ULOS/Modulo_I_.pdf

Anderson, T., \& Darlin, D. (1952). Asymptotic Theory of Certain "Goodness of Fit" Criteria Based on Stochastic Processes. The Annals of Mathematical Statistics, 23(2), 193-212.

Ang, A., \& Willson, T. (2007). Probability Concepts in Engineering. Emphasis on Applications on Civil and Eviromental Engineering. USA: Johm Wiley and Sons, Inc.

Arroyo, I., Bravo, L., Llinás, H., \& Muñoz, F. (2014). Distribuciones Poisson y Gamma: Una discreta y continua relación. Prospect 12(1), 99-107. Recuperado de http://www.scielo.org.co/pdf/prosp/v12n1/v12n1a12.pdf

Bacaria, J., Osorio, M., \& Artal, A. (s.f.). Evaluación del Acuerdo de Libre Comercio México - Unión Europea.

Banco Interamericano de Desarrollo (2010). La era de la productividad: cómo transformar las economías desde sus cimientos. Desarrollo en las Américas (DIA) 2010. Chile: BID - Fondo de Cultura Económica. Recuperado de https://publications.iadb.org/handle/11319/4478?locale-attribute=es

Blyde, J. (2014). Fábricas Sincronizadas: América Latina y el Caribe en la Era de las Cadenas Globales de Valor. USA: Banco Interamericano de Desarrollo. Recuperado de https://publications.iadb.org/handle/11319/6668

Cruz, J. (03 de 04 de 2017). Sitio del Instituto Mexicano de Ejecutivos en Finanzas IMEF. Recuperado de Conferencia IMEF. Capítulo 1. Desempeño Histórico 1914-2004. Ponencia: Estabilidad y Crecimiento. Instituto Mexicano de Ejecutivos de Finanzas: http://imef.org.mx/ponencia/2005/PONENCIAIMEF.pdf

Curzio, L. (2004). La integración en Norteamérica y la experiencia de Europa. En Roy, J., Chanona, A., \& Domínguez, R. La Unión Europea y el TLCAN: Integración Regional Comparada y Relaciones Mutuas. (Primera ed., pp. 159-182). México: UNAM.

Franco, A. (2016). México en 2030: Superando la Referencia. América Latina y el Caribe 2030: Escenarios futuros. Atlantic Council / BID / Centro Frederick S. Pardee for International Futures. USA: Banco Interamericano de Desarrollo. Recuperado de https://publications.iadb.org/handle/11319/7978 
Frontini, G., \& Bonnefoy, P. (03 de 03 de 2017). Relaciones comerciales entre la UE y México. En e. Solana, México: un destino natural y estratégico (págs. 101-120). España: Santander - Universidad de Nebrija. Recuperado de http://biblioteca.cunef.edu/gestion/catalogo/doc_num.php?explnum_id=1183

Giordano, P., \& Ramos, A. (2016). Cambio de marcha: América Latina y el Caribe en la nueva normalidad del comercio. "Monitor de Comercio e Integración 2016”. USA: Banco Interamericano de Desarrollo. Recuperado de https://publications.iadb.org/bitstream/handle/11319/7942/Monitor-deComercio-e-Integracion-2016-Cambio-de-marcha-America-Latina-y-el-Caribeen-la-nueva-normalidad-del-comercio-global.pdf

International Trade Centre. (28 de 03 de 2017). Trade Map - International Trade Statistics. Recuperado de http://www.trademap.org/tradestat/Country_SelProductCountry_TS.aspx?nvpm =3|484||||TOTAL ||$|2| 1|1| 2|2| 1|2| 1 \mid 1$

Jenkins, R. (2016). Estado del arte sobre las relaciones con China. Made in CHI-LAT: Claves para renovar la convergencia entre Latinoamérica y China. Integración y Comercio. USA: INTAL - Banco Interamericano de Desarrollo.

Khodabin, M., \& Ahmadabadi, A. (2010). Some properties of generalized gamma distribution. Mathematical Sciences, 4(1), 9-28. Recuperado de http://www.sid.ir/EN/VEWSSID/J_pdf/1010720100102.pdf.

Lora, E., \& Pagés, C. (2011). La Realidad Macroeconómica. Módulo 2. USA: Banco Interamericano de Desarrollo. Recuperado de https://indesvirtual.iadb.org/pluginfile.php/45726/mod_resource/content/0/MOD ULOS/Modulo_II.pdf

Marczak, J., Engelke, P., Bohl, D., \& Saldarriaga, A. (2016). América Latina y el Caribe 2030: Escenarios futuros. USA: Atlantic Council/BID/Centro Frederick S. Pardee for International Futures. Recuperado de https://C:/Users/74999/Downloads/LAC2030-America-Latina-y-el-Caribe2030-Escenarios-futuros.pdf

Monserrat, H., \& Chávez, M.F. (2003). Tres modelos de política económica en México durante los últimos sesenta años. Análisis Económico, XVIII(37), 55-80.

Myers, M. (2016). Una nueva era de reformas. Made in CHI-LAT: Claves para renovar la convergencia entre Latinoamérica y China. Integración y Comercio. USA: INTAL - Banco Interamericano de Desarrollo.

Ortiz, A. (2014). La política comercial de México a lo largo de dos décadas del TLCAN: una visión crítica. En A. Ortíz, TLCAN 20 años: ¿Celebración, desencanto o replanteamiento? (pp. 133-155). México: México: UNAM / Instituto de Investigaciones Jurídicas. 
Pastor, R. (2014). El futuro de México en América del Norte: su tercer desafío. En A. (. Oropeza, TLCAN 20 años ¿Celebración, desencanto o replanteamiento? (Primera ed., pp. 357-374). México: UNAM / Instituto de Investigaciones Jurídicas.

Pomory, C. (2006). A note on calculating P values from 0.15-0.005 for the AndersonDarling normality test using the F distribution. Journal of Applied Statistics, 33(4), 461-462. Recuperado de http://www.tandfonline.com/doi/full/10.1080/02664760600677720

PROMEXICO. (16 de 03 de 2017). PROMEXICO Comunicación. Recuperado de http://www.promexico.mx/documentos/infograficos/mexico-in-the-world2015.pdf

Ramírez, J. (2014). La competencia Estados Unidos-China: El Trans-Pacific Partnership Agreement vs el Acuerdo de Libre Comercio China-Corea-Japón. Políticas Institucionales de Integración Regional. México: El Colegio de México.

Sehgal, S. (2010). The Evolution of NAFTA. India Quarterly: A Journal of International Affairs, 66(3), 303-316. Recuperado de http://journals.sagepub.com/doi/pdf/10.1177/097492841006600305

Shearer, M., \& Tres, J. (2016). Nuevas tendencias en los tratados comerciales en América Latina. Módulo I. ¿Qué son los acuerdos comerciales y cuál es su importancia? USA: Banco Interamericano de Desarrollo.

Stephens, M. (1979a). The Anderson-Darling Statistic. Stanford, USA: Department of Statistics, Stanford University. Recuperado de http://www.dtic.mil/dtic/tr/fulltext/u2/a079807.pdf

Stephens, M. (1979b). The Anderson-Darling Statistic. Technical Report Num. 39. USA: Department of Statistics, Stanford University.

Thom, J. (1958). A note on the gamma distribution. Monthly Weather Review, 86(4), 117122.

Torres, B., \& Vega, G. (2010). Los Grandes Problemas de México Vol. XII Relaciones Internacionales. México: El Colegio de México.

Vega, G. (2011). El Tratado de Libre Comercio México Unión Europea (TLCUEM) Una evaluación a diez años. En E. Tremolada, ¿Europa: un marco de oportunidades? (pp. 127-178). Colombia: Universidad Externado de Colombia.

Vega, G. (2014). México y Estados Unidos. La Economía Política del Libre Comercio. Antología, 1. México: El Colegio de México.

Villareal, R. (2004). México en su laberinto ¿Cómo replantear el modelo económico en una era post TLCAN? En TLCAN 20 años ¿Celebración, Desencanto o Replanteamiento? México: UNAM. 
Wackerly, D., Mendenhall, W., \& Scheafer, R.L. (2010). Estadística Matemática con aplicaciones. México: Cengage Learning. 